\title{
An analysis of the L1 scheme for stochastic subdiffusion problem driven by integrated space-time white noise
}

\author{
Xiaolei Wu $\mathrm{Wu}^{\mathrm{a}}$, Yuyan Yan ${ }^{\mathrm{b}}$, Yubin Yan*,c \\ ${ }^{a}$ Department of Mathematics, Lvliang University, Lishi, P.R.China, 033000 \\ ${ }^{b}$ School of Sciences, Jimei University, Xiamen 361021, China \\ ${ }^{c}$ Department of Mathematics, University of Chester, CH1 $4 \mathrm{BJ}$
}

\begin{abstract}
We consider the strong convergence of the numerical methods for solving stochastic subdiffusion problem driven by an integrated space-time white noise. The time fractional derivative is approximated by using the L1 scheme and the time fractional integral is approximated with the Lubich's first order convolution quadrature formula. We use the Euler method to approximate the noise in time and use the truncated series to approximate the noise in space. The spatial variable is discretized by using the linear finite element method. Applying the idea in Gunzburger et al. (Math. Comp. 88(2019), pp. 1715-1741), we express the approximate solutions of the fully discrete scheme by the convolution of the piecewise constant function and the inverse Laplace transform of the resolvent related function. Based on such convolution expressions of the approximate solutions, we obtain the optimal convergence orders of the fully discrete scheme in spatial multi-dimensional cases by using the Laplace transform method and the corresponding resolvent estimates.
\end{abstract}

Key words:

Fractional derivative, stochastic subdiffusion, finite element method, error estimates AMS Subject Classification: 65M12; 65M06; 65M70; $35 S 10$

\section{Introduction}

In this paper, we will consider the numerical methods for solving the following stochastic time-fractional partial differential equation driven by integrated noise, with $\alpha \in(0,1), \gamma \in[0,1]$,

$$
{ }_{0}^{C} D_{t}^{\alpha} u(t)+A u(t)={ }_{0}^{R} D_{t}^{-\gamma} \frac{d W(t)}{d t}, \quad \text { for } 0<t \leq T, \quad \text { with } u(0)=u_{0},
$$

where $A: \mathcal{D}(A) \rightarrow H$ is an elliptic operator, with $\mathcal{D}(A)=H_{0}^{1}(\mathcal{D}) \cap H^{2}(\mathcal{D})$ and $\mathcal{D} \subset \mathbb{R}^{d}, d=1,2,3$ is some regular domain. For example, we shall consider $A=-\Delta$ with $\Delta$ the Laplacian and $H_{0}^{1}(\mathcal{D}) \cap H^{2}(\mathcal{D})$ denotes the standard Sobolev spaces. Here ${ }_{0}^{C} D_{t}^{\alpha} u(t)$ denotes the Caputo fractional derivative defined by, with $u^{\prime}(s)=\frac{d u}{d s}$,

$$
{ }_{0}^{C} D_{t}^{\alpha} u(t)=\frac{1}{\Gamma(1-\alpha)} \int_{0}^{t}(t-s)^{-\alpha} u^{\prime}(s) d s,
$$

and ${ }_{0}^{R} D_{t}^{-\gamma} u(t)$ denotes the Riemann-Liouville fractional integral defined by

$$
{ }_{0}^{R} D_{t}^{-\gamma} u(t)=\frac{1}{\Gamma(\gamma)} \int_{0}^{t}(t-s)^{\gamma-1} u(s) d s .
$$

\footnotetext{
* Corresponding author

Email addresses: www (Xiaolei Wu), XYuyuan@163.com (Yuyuan Yan), y.yan@chester.ac.uk (Yubin Yan)
} 
Since we are mainly interested in the error estimates induced by the stochastic perturbation, for simplicity, we shall assume the initial value $u_{0}=0$ in this work. In such case, the Caputo fractional derivative ${ }_{0}^{C} D_{t}^{\alpha} u(t), \alpha \in(0,1)$ is the same as the Riemann-Liouville fractional derivative ${ }_{0}^{R} D_{t}^{\alpha} u(t), \alpha \in(0,1)$.

For the elliptic operator $A$, we may assume that it satisfies the following resolvent estimate, with $\theta \in$ $(\pi / 2, \pi)$, see Lubich et al. [27], Thomée [38],

$$
\left\|(z I+A)^{-1}\right\| \leq C|z|^{-1} \quad \text { for } z \in \Sigma_{\theta}=\{z \neq 0:|\arg \mathrm{z}|<\theta\},
$$

which implies that, with $\alpha \in(0,1)$, see Yan et al. [36],

$$
\left\|\left(z^{\alpha} I+A\right)^{-1}\right\| \leq C|z|^{-\alpha}, \quad \forall z \in \Sigma_{\theta}=\{z \neq 0:|\arg \mathrm{z}|<\theta\} .
$$

For example, we may choose $A=-\Delta, \mathcal{D}(A)=H_{0}^{1}(\mathcal{D}) \cap H^{2}(\mathcal{D})$, where $\Delta$ denotes the Laplacian operator. For the noise term $\frac{d W(t)}{d t}$, we assume that $W(t)$ takes the following Fourier series form

$$
W(t)=\sum_{j=1}^{\infty} e_{j} \beta_{j}(t),
$$

where $\beta_{j}(t), j=1,2, \ldots$ denote the independent, identically distributed Brownian motions and $\left\{\lambda_{j}, e_{j}\right\}_{j=1}^{\infty}$ are the eigenpairs of the elliptic operator $A$.

Many application problems can be modeled by (1), for example, thermal diffusion in media with fractional geometry [32], highly heterogeneous aquifer [1], underground environmental problems [16], random walks [13], etc.

The existence, uniqueness and regularity of the deterministic time fractional partial differential equations have been studied extensively, see Sakamoto and Yamamoto [33] and Jin et al. [17] and the references therein. There are also many numerical methods for solving deterministic time fractional partial differential equation, see, e.g., [25], [12], [41], [11], [10], [37], [17], [36], [20], [28], [31], [40], [42], [7], [34], [35], etc.

The SPDEs with fractional time derivative that we are going to study in this paper naturally arise from the consideration of the heat equation in a material with thermal memory. The purpose of introducing the noise term $W(t)$ in the stochastic model (1) is to describe random effects on transport of particles in medium with memory or particles subject to sticking and trapping [5]. Recently, there is increasing interest for the studies of equation (1). Chen et al. [5] proved the existence and uniqueness of a solution to a stochastic time-fractional PDE in both divergence and non-divergence forms. Mijena and Nane [29] showed the existence and uniqueness of a continuous random field solution to a stochastic space-timefractional PDE, and more recently they [30] studied weak intermittency of the solution and the propagation of intermittency front. Chen [3] analyzed the moments, Hölder continuity and intermittency of the solution for one-dimensional nonlinear stochastic time-fractional diffusion, see also [9, 4]. Liu et al. [26] considered the existence and uniqueness of the solution to (1) with the more general quasi-linear elliptic operator. Anh et al. [2] investigated the weak-sense solution of a fractional-in-space and in-time stochastic PDE.

Let us review some numerical methods for solving stochastic fractional partial differential equation. Kovács and Printems [22], [23] considerd the strong and weak convergence for solving stochastic volterra intergal partial differential equation. Li et al. [24] considered the finite element method for solving stochastic super-diffusion equation. Jin et al. [21] considered the numerical methods for stochastic time fractional partial differential equation driven by integrated noise.

More recently, Gunzburger et al. [14], [15] considered the time discretization and the finite element methods for solving stochastic integral-differential equations driven by space-time white noise. The time fractional Riemann-Liouville derivative and the time fractional integral were approximated by using the first order convolution quadrature formula. The approximate solution was expressed by using the convolution of the piecewise constant function and the inverse Laplace transform of the resolvent related function and the optimal convergence orders in multidimensional case were proved by using the Laplace transform method and the resolvent estimates.

In this paper, we shall consider the numerical methods for solving stochastic time fractional partial differential equation driven by integrated space-time white noise. We approximate the Caputo time fractional 
derivative by using the L1 scheme and approximate the Riemann-Liouville fractional integral by using the first order Lubich convolution quadrature formula. The space-time white noise is approximated by using the Euler method in time and the truncated series in space. The spatial variable is approximated with a standard finite element method. The approximate solution is expressed with the convolution of the piecewise constant function and the inverse Laplace transform of the resolvent related function. Based on such convolution expression of the approximate solution, we obtain the optimal error estimates in $L^{2}\left(\Omega ; L^{2}(\mathcal{D})\right)$ norm by using the Laplace transform method. Here $\Omega$ denotes the sample probability space.

The main contributions of the paper are as follows:

1. An L1 scheme for solving stochastic time-fractional partial differential equations is introduced.

2. A new expression of the approximate solution for stochastic time-fractional partial differential equation is developed which is based on the convolution of the piecewise constant function and the inverse Laplace transform of the resolvent related function.

3. The optimal error estimates for solving stochastic time fractional partial differential equations are proved for high spacial dimensional problem with $\mathcal{D} \subset \mathbb{R}^{d}, d=1,2,3$ and for both the white and the trace class noises.

The paper is organized as follows. In Section 2, we consider the time discretization and the optimal convergence rates are obtained in multidimensional case with respect to the time step size. In Section 3, we consider the finite element method and the optimal convergence rates are obtained in multidimensional case with respect to the space step size.

By $C$ we denote a positive constant independent of the functions and parameters concerned, but not necessarily the same at different occurrences. By $c$ we denote a particular positive constant independent of the functions and parameters concerned.

\section{Time discretization}

In this section, we shall consider the time discretization of $(1)$. Denote $f(t)=\frac{d W(t)}{d t}$. Then $(1)$ can be written as

$$
{ }_{0}^{C} D_{t}^{\alpha} u(t)+A u(t)={ }_{0}^{R} D_{t}^{-\gamma} f(t), \quad \text { for } 0<t \leq T, \quad \text { with } u(0)=0,
$$

Taking the Laplace transform of (5), we have

$$
z^{\alpha} \hat{u}(z)+A \hat{u}(z)=z^{-\gamma} \hat{f}(z)
$$

which implies that

$$
\hat{u}(z)=\left(z^{\alpha}+A\right)^{-1} z^{-\gamma} \hat{f}(z) .
$$

By the inverse Laplace transform, we have, see Yan et al. [36],

$$
u(t)=\frac{1}{2 \pi i} \int_{\Gamma} e^{z t}\left(z^{\alpha}+A\right)^{-1} z^{-\gamma} \hat{f}(z) d z,
$$

where $\Gamma=\{z: \arg (z)=\theta, \theta \in(\pi / 2, \pi), \Im(z)$ increases from $-\infty$ to $\infty\}$.

Let $\left(z^{\alpha}+A\right)^{-1} z^{-\gamma}$ be the Laplace transform of $E(t)$, i.e., $\widehat{E}(z)=\left(z^{\alpha}+A\right)^{-1} z^{-\gamma}$, we then have

$$
E(t)=\frac{1}{2 \pi i} \int_{\Gamma} e^{z t} \hat{E}(z) d z=\frac{1}{2 \pi i} \int_{\Gamma} e^{z t}\left(z^{\alpha}+A\right)^{-1} z^{-\gamma} d z
$$

Hence the solution of (5) can be written as

$$
u(t)=(E * f)(t)=\int_{0}^{t} E(t-s) f(s) d s .
$$


Let $0=t_{0}<t_{1}<t_{2}<\cdots<t_{N}=T$ be a partition of $[0, T]$ and $\tau$ the time step size. We shall approximate the time fractional derivative at $t=t_{n}$ by using the L1 scheme, with $0<\alpha<1$,

$$
{ }_{0}^{C} D_{t}^{\alpha} u\left(t_{n}\right)=\tau^{-\alpha} \sum_{k=0}^{n} w_{n-k}^{(\alpha)} u\left(t_{k}\right)+O\left(\tau^{2-\alpha}\right),
$$

where $w_{k}^{(\alpha)}, k=1,2, \ldots, n$ are defined by, see Jin et al. [18] and Yan et al. [36],

$$
\Gamma(2-\alpha) w_{k}^{(\alpha)}=\left\{\begin{array}{l}
1, \quad \text { for } k=0, \\
-2 k^{1-\alpha}+(k-1)^{1-\alpha}+(k+1)^{1-\alpha}, \quad \text { for } k=1,2, \ldots, n-1 \\
(k-1)^{1-\alpha}-k^{1-\alpha}, \quad \text { for } k=n .
\end{array}\right.
$$

Further we approximate the Riemann-Liouville fractional integral by the first order convolution quadrature formula, that is,

$$
{ }_{0}^{R} D_{t}^{-\gamma} u\left(t_{n}\right)=\tau^{\gamma} \sum_{k=0}^{n} w_{n-k}^{(\gamma)} u\left(t_{k}\right)+O(\tau)
$$

where $w_{k}^{(\gamma)}, k=0,1, \ldots$ are generated by $(1-\zeta)^{-\gamma}$, that is, see Jin et al. [20],

$$
(1-\zeta)^{-\gamma}=\sum_{k=0}^{\infty} w_{k}^{(\gamma)} \zeta^{k}
$$

For the noise $\frac{d W(t)}{d t}$ term, we approximate it at $t=t_{n}$ by using the Euler method, that is,

$$
\frac{d W\left(t_{n}\right)}{d t} \approx\left(W\left(t_{n}\right)-W\left(t_{n-1}\right)\right) / \tau
$$

Let $u^{n} \approx u\left(t_{n}\right)$ denote the approximate solution of $u\left(t_{n}\right)$. We define the following time discretization scheme for solving (5): with $f^{n}=\left(W\left(t_{n}\right)-W\left(t_{n-1}\right)\right) / \tau, n=1,2, \ldots$ and $f^{0}=0$,

$$
\tau^{-\alpha} \sum_{k=0}^{n} w_{n-k}^{(\alpha)} u^{k}+A u^{n}=\tau^{\gamma} \sum_{k=0}^{n} w_{n-k}^{(\gamma)} f^{k}, n=1,2, \ldots, \quad \text { with } u^{0}=0 .
$$

Taking the discrete Laplace transform in both sides of (7), we have

$$
\sum_{n=1}^{\infty}\left(\tau^{-\alpha} \sum_{k=0}^{n} w_{n-k}^{(\alpha)} u^{k}\right) \zeta^{n}+\sum_{n=1}^{\infty}\left(A u^{n}\right) \zeta^{n}=\sum_{n=1}^{\infty}\left(\tau^{\gamma} \sum_{k=0}^{n} w_{n-k}^{(\gamma)} f^{k}\right) \zeta^{n}
$$

Denote the discrete Laplace transforms of the sequences $\left\{w_{n}^{(\alpha)}\right\}_{n=0}^{\infty},\left\{w_{n}^{(\gamma)}\right\}_{n=0}^{\infty}$ and $\left\{u^{n}\right\}_{n=0}^{\infty}$ by

$$
\widetilde{w}^{(\alpha)}(\zeta)=\sum_{n=0}^{\infty} w_{n}^{(\alpha)} \zeta^{n}, \quad \widetilde{w}^{(\gamma)}(\zeta)=\sum_{n=0}^{\infty} w_{n}^{(\gamma)} \zeta^{n}, \quad \widetilde{u}(\zeta)=\sum_{n=0}^{\infty} u^{n} \zeta^{n}
$$

respectively. We then have, with $\tilde{f}(\zeta)=\sum_{n=0}^{\infty} f^{n} \zeta^{n}=\sum_{n=1}^{\infty} f^{n} \zeta^{n}$,

$$
\tau^{-\alpha} \widetilde{w}^{(\alpha)}(\zeta) \widetilde{u}(\zeta)+A \widetilde{u}(\zeta)=\tau^{\gamma} \widetilde{w}^{(\gamma)}(\zeta) \tilde{f}(\zeta),
$$

which implies that

$$
\widetilde{u}(\zeta)=\left(\tau^{-\alpha} \widetilde{w}^{(\alpha)}(\zeta)+A\right)^{-1}\left(\tau^{\gamma} \widetilde{w}^{(\gamma)}(\zeta) \tilde{f}(\zeta)\right)
$$


By using the inverse discrete Laplace transform, we have, with $\zeta=e^{-\tau z}$, see, [36],

$$
\begin{aligned}
u^{n} & =\frac{\tau}{2 \pi i} \int_{\Gamma_{\tau}} e^{z t_{n}}\left(\tau^{-\alpha} \widetilde{w}^{(\alpha)}(\zeta)+A\right)^{-1}\left(\tau^{\gamma} \widetilde{w}^{(\gamma)}(\zeta) \tilde{f}(\zeta)\right) d z \\
& =\frac{\tau}{2 \pi i} \int_{\Gamma_{\tau}} e^{z t_{n}}\left(\tau^{-\alpha} \widetilde{w}^{(\alpha)}\left(e^{-\tau z}\right)+A\right)^{-1}\left(\tau^{\gamma} \widetilde{w}^{(\gamma)}\left(e^{-\tau z}\right) \tilde{f}\left(e^{-\tau z}\right)\right)
\end{aligned}
$$

where $\Gamma_{\tau}=\left\{z: z \in \Gamma,|\Im z| \leq \frac{\pi}{\tau}\right\}$.

Denote $z_{1}$ and $z_{2}$ by

$$
z_{1}=\tau^{-1}\left(\widetilde{w}^{(\alpha)}\left(e^{-\tau z}\right)\right)^{1 / \alpha} \quad \text { and } \quad z_{2}=\tau^{-1}\left(1-e^{-\tau z}\right)
$$

where $z_{1}$ and $z_{2}$ are some suitable approximations of $z \in \Gamma_{\tau}$. We then have

$$
z_{1}^{\alpha}=\tau^{-\alpha} \widetilde{w}^{(\alpha)}\left(e^{-\tau z}\right), \quad \text { and } z_{2}^{-\gamma}=\tau^{\gamma}\left(1-e^{-\tau z}\right)^{-\gamma} .
$$

Thus $u^{n}$ can be written as

$$
u^{n}=\frac{\tau}{2 \pi i} \int_{\Gamma_{\tau}} e^{z t_{n}}\left(z_{1}^{\alpha}+A\right)^{-1} z_{2}^{-\gamma} \tilde{f}\left(e^{-\tau z}\right) d z .
$$

We will prove that, in Lemma 2.1 below, $u^{n}$ can be expressed as the convolution of the piecewise constant function $\bar{f}(t)$ defined in (12) below and the inverse Laplace transform of the resolvent related function $E_{\tau}(t)$ defined in (14) below. To see this, we first introduce the following piecewise constant function $\bar{f}(t), t>0$ defined by

$$
\bar{f}(t)=\left\{\begin{array}{l}
f^{n}, t \in\left(t_{n-1}, t_{n}\right], n=1,2, \ldots, N, \\
0, \quad t>T=t_{N} .
\end{array}\right.
$$

We now show that $u^{n}$ in (7) or (11) can be written as

$$
u^{n}=\frac{\tau}{2 \pi i} \int_{\Gamma_{\tau}} e^{z t_{n}}\left(z_{1}^{\alpha}+A\right)^{-1} z_{2}^{-\gamma} \frac{z}{e^{z \tau}-1} \hat{\bar{f}}(z) d z,
$$

where $\hat{\bar{f}}(z)$ denotes the Laplace transform of $\bar{f}(t)$. In fact, we have

$$
\begin{aligned}
& \frac{\tau}{2 \pi i} \int_{\Gamma_{\tau}} e^{z t_{n}}\left(z_{1}^{\alpha}+A\right)^{-1} z_{2}^{-\gamma} \frac{z}{e^{z \tau}-1} \hat{\bar{f}}(z) d z \\
& \quad=\frac{\tau}{2 \pi i} \int_{\Gamma_{\tau}} e^{z t_{n}}\left(z_{1}^{\alpha}+A\right)^{-1} z_{2}^{-\gamma} \frac{z}{e^{z \tau}-1}\left(\int_{0}^{\infty} \bar{f}(t) e^{-t z} d t\right) d z \\
& \quad=\frac{\tau}{2 \pi i} \int_{\Gamma_{\tau}} e^{z t_{n}}\left(z_{1}^{\alpha}+A\right)^{-1} z_{2}^{-\gamma} \frac{z}{e^{z \tau}-1}\left(\sum_{n=1}^{\infty} \int_{t_{n-1}}^{t_{n}} f^{n} e^{-t z} d t\right) d z \\
& =\frac{\tau}{2 \pi i} \int_{\Gamma_{\tau}} e^{z t_{n}}\left(z_{1}^{\alpha}+A\right)^{-1} z_{2}^{-\gamma} \frac{z}{e^{z \tau}-1}\left(\sum_{n=1}^{\infty} f^{n}\left(\frac{e^{-t_{n-1} z}-e^{-t_{n} z}}{z}\right) d z\right. \\
& \quad=\frac{\tau}{2 \pi i} \int_{\Gamma_{\tau}} e^{z t_{n}}\left(z_{1}^{\alpha}+A\right)^{-1} z_{2}^{-\gamma}\left(\sum_{n=1}^{\infty} f^{n} e^{-t_{n} z}\right) d z
\end{aligned}
$$

Note that $\tilde{f}(\zeta)=\sum_{n=0}^{\infty} f^{n} \zeta^{n}=\sum_{n=1}^{\infty} f^{n} \zeta^{n}$, where $f^{n}=\left(W\left(t_{n}\right)-W\left(t_{n-1}\right)\right) / \tau, n=1,2, \ldots$ and $f^{0}=0$, we have

$$
\sum_{n=1}^{\infty} f^{n} e^{-t_{n} z}=\sum_{n=1}^{\infty} f^{n}\left(e^{-\tau z}\right)^{n}=\tilde{f}\left(e^{-\tau z}\right) .
$$

Thus we get, by (11),

$$
\frac{\tau}{2 \pi i} \int_{\Gamma_{\tau}} e^{z t_{n}}\left(z_{1}^{\alpha}+A\right)^{-1} z_{2}^{-\gamma} \frac{z}{e^{z \tau}-1} \hat{\bar{f}}(z) d z=\frac{\tau}{2 \pi i} \int_{\Gamma_{\tau}} e^{z t_{n}}\left(z_{1}^{\alpha}+A\right)^{-1} z_{2}^{-\gamma} \tilde{f}\left(e^{-\tau z}\right) d z=u^{n}
$$


which completes the proof of (13).

Denote

$$
E_{\tau}(t)=\frac{\tau}{2 \pi i} \int_{\Gamma_{\tau}} e^{z t}\left(z_{1}^{\alpha}+A\right)^{-1} z_{2}^{-\gamma} \frac{z}{e^{z \tau}-1} d z,
$$

we have the following lemma:

Lemma 2.1. The solution $u^{n}$ of (7) has the following form

$$
u^{n}=\int_{0}^{t_{n}} E_{\tau}\left(t_{n}-s\right) \bar{f}(s) d s,
$$

where $E_{\tau}(t)$ and $\bar{f}(t)$ are defined by (14) and (12), respectively.

Proof: Since $u^{n}$ depends only on $f\left(t_{j}\right)$ for $j \leq n$, we may simply set $\bar{f}(t)=0$ for $t>t_{n}$ below.

Denote

$$
v(t)=\int_{0}^{t} E_{\tau}(t-s) \bar{f}(s) d s .
$$

It suffices to show that

$$
u^{n}=v\left(t_{n}\right),
$$

which we will prove it now.

Taking the Laplace transform in (15), we get

$$
\hat{v}(\xi)=\widehat{E}_{\tau}(\xi) \hat{\bar{f}}(\xi), \quad \forall \xi \in \Gamma,
$$

where, with $z \in \Gamma_{\tau}$,

$$
\begin{aligned}
\widehat{E}_{\tau}(\xi) & =\int_{0}^{\infty} e^{-\xi t}\left(\frac{\tau}{2 \pi i} \int_{\Gamma_{\tau}} e^{z t}\left(z_{1}^{\alpha}+A\right)^{-1} z_{2}^{-\gamma} \frac{z}{e^{\tau z}-1} d z\right) d t \\
& \left.=\frac{\tau}{2 \pi i} \int_{\Gamma_{\tau}}\left(\int_{0}^{\infty} e^{-(\xi-z) t} d t\right)\left(z_{1}^{\alpha}+A\right)^{-1} z_{2}^{-\gamma} \frac{z}{e^{\tau z}-1} d z\right) \\
& =\frac{\tau}{2 \pi i} \int_{\Gamma_{\tau}} \frac{1}{\xi-z}\left(z_{1}^{\alpha}+A\right)^{-1} z_{2}^{-\gamma} \frac{z}{e^{\tau z}-1} d z
\end{aligned}
$$

Thus we have

$$
\begin{aligned}
v\left(t_{n}\right) & =\frac{1}{2 \pi i} \int_{\Gamma} e^{\xi t_{n}} \hat{v}(\xi) d \xi=\frac{1}{2 \pi i} \int_{\Gamma} e^{\xi t_{n}} \hat{E}_{\tau}(\xi) \hat{\bar{f}}(\xi) d \xi \\
& =\frac{1}{2 \pi i} \int_{\Gamma} e^{\xi t_{n}}\left(\frac{\tau}{2 \pi i} \int_{\Gamma_{\tau}} \frac{1}{\xi-z}\left(z_{1}^{\alpha}+A\right)^{-1} z_{2}^{-\gamma} \frac{z}{e^{\tau z}-1} d z\right) \hat{f}(\xi) d \xi \\
& =\frac{\tau}{2 \pi i} \int_{\Gamma_{\tau}}\left(z_{1}^{\alpha}+A\right)^{-1} z_{2}^{-\gamma} \frac{z}{e^{\tau z}-1}\left(\frac{1}{2 \pi i} \int_{\Gamma} e^{\xi t_{n}} \frac{1}{\xi-z} \hat{\bar{f}}(\xi) d \xi\right) d z .
\end{aligned}
$$

By the Cauchy integral formula, we have

$$
\begin{aligned}
& \frac{1}{2 \pi i} \int_{\Gamma} e^{\xi t_{n}} \frac{1}{\xi-z} \hat{\bar{f}}(\xi) d \xi=\frac{1}{2 \pi i} \int_{\Gamma} e^{\xi t_{n}} \frac{1}{\xi-z}\left(\int_{0}^{\infty} e^{-\xi t} \bar{f}(t) d t\right) d \xi \\
= & \frac{1}{2 \pi i} \int_{\Gamma} e^{\xi t_{n}} \frac{1}{\xi-z}\left(\int_{0}^{t_{n}} e^{-\xi t} \bar{f}(t) d t\right) d \xi=\int_{0}^{t_{n}}\left(\frac{1}{2 \pi i} \int_{\Gamma} e^{\xi\left(t_{n}-t\right)} \frac{1}{\xi-z} d \xi\right) \bar{f}(t) d t \\
= & \int_{0}^{t_{n}} e^{z\left(t_{n}-t\right)} \bar{f}(t) d t=e^{z t_{n}} \hat{f}(z) .
\end{aligned}
$$


Thus we have

$$
v\left(t_{n}\right)=\frac{\tau}{2 \pi i} \int_{\Gamma_{\tau}} e^{z t_{n}}\left(z_{1}^{\alpha}+A\right)^{-1} z_{2}^{-\gamma} \frac{z}{e^{z \tau}-1} \hat{\hat{f}}(z) d z,
$$

which indeed is $u^{n}$ by (13).

Together these estimates complete the proof of Lemma 2.1.

Let us now introduce the Isometry property Lemma which we will use frequently in the proof of the error estimates below. Let $L(H)$ be the space of bounded linear operators from $H$ to $H$, and $L_{2}^{0}=$ $H S\left(Q^{1 / 2}(H), H\right)$ be the space of Hilbert-Schmidt operators from $Q^{1 / 2}(H)$ to $H$, i.e.,

$$
L_{2}^{0}=\left\{\psi \in L(H): \sum_{l=1}^{\infty}\left\|\psi Q^{1 / 2} e_{l}\right\|^{2}<\infty\right\}
$$

with the norm $\|\cdot\|_{L_{2}^{0}}$ given by

$$
\|\psi\|_{L_{2}^{0}}^{2}=\sum_{l=1}^{\infty}\left\|\psi Q^{1 / 2} e_{l}\right\|^{2} .
$$

Let $\mathbb{E}$ denote the expectation. For $\psi \in L_{2}^{0}$, the integral $\int_{0}^{t} \psi(s) d W(s)$ is well defined in the sense of stochastic integral [6, pp. 95] and the following isometry properties hold [6, pp. 101]:

Lemma 2.2. We have

$$
\mathbb{E}\left\|\int_{0}^{t} \psi(s) d W(s)\right\|^{2}=\int_{0}^{t} \mathbb{E}\|\psi(s)\|_{L_{2}^{0}}^{2} d s,
$$

and in the the discrete form

$$
\mathbb{E}\left\|\sum_{i=1}^{n} \int_{t_{i-1}}^{t_{i}} \psi(s) d W(s)\right\|^{2}=\sum_{i=1}^{n} \int_{t_{i-1}}^{t_{i}} \mathbb{E}\|\psi(s)\|_{L_{2}^{0}}^{2} d s .
$$

We next introduce some lemmas which we also need in the proofs of Theorems 2.8 and 3.1 .

Lemma 2.3. Let $\lambda>0$, we have

$$
\left|(z+\lambda)^{-1}\right| \leq C(|z|+\lambda)^{-1}, \quad \forall z \in \Sigma_{\varphi}=\{z:|\arg z| \leq \varphi, \varphi \in(0, \pi)\}
$$

Proof: The proof of this lemma was given in [14, Lemma 3.3]. For completeness, we give the sketch of the proof here.

Let $\xi=-\lambda+0 i$. We have

$$
|z-\xi|=|z+\lambda|
$$

For simplicity, we only consider the case $\varphi \in(\pi / 2, \pi)$. We now consider the triangle with three vertices $z, 0$ and $\xi$ with interior angles $w_{z}, w_{0}$ and $w_{\xi}$ at the three vertices, respectively. Assume that $w_{0} \geq \pi / 2$, we then have

$$
|z-\xi| \geq|z|, \quad \text { and }|z-\xi| \geq|\xi|=\lambda
$$

which implies that

$$
|z-\xi| \geq \frac{1}{2}(|z|+\lambda)
$$

Hence (21) follows.

We now consider the case for $w_{0}<\pi / 2$. Note that

$$
\frac{|z-\xi|}{\sin w_{0}}=\frac{|z|}{\sin w_{\xi}}=\frac{\lambda}{\sin w_{z}} .
$$


We then have, since $w_{0} \geq \pi-\varphi$,

$$
|z-\xi|=\frac{\lambda \sin w_{0}}{\sin w_{\xi}} \geq \frac{|z| \sin (\pi-\varphi)}{\sin w_{\xi}} \geq|z| \sin \varphi
$$

and

which implies that

$$
|z-\xi|=\frac{\lambda \sin w_{0}}{\sin w_{z}} \geq \frac{\lambda \sin (\pi-\varphi)}{\sin w_{z}} \geq \lambda \sin \varphi
$$

$$
|z+\lambda|=|z-\xi| \geq \frac{\sin \varphi}{2}(|z|+\lambda) .
$$

Together these estimates complete the proof of Lemma 2.3 .

Lemma 2.4. We have

$$
\left\|\left(z^{\alpha}+A\right)^{-1}\right\|_{H S} \leq C|z|^{-\alpha}|z|^{\alpha d / 4}, \quad \forall z \in \Sigma_{\varphi} \text { with } \varphi \in(0, \pi) .
$$

Proof: We will consider two cases for $|z|>1$ and $0<|z| \leq 1$.

For the case $|z|>1$, we have, by using Lemma 2.3, with some $s>0$ determined later,

$$
\begin{aligned}
\left\|\left(z^{\alpha}+A\right)^{-1}\right\|_{H S}^{2} & =\sum_{j=1}^{\infty}\left(\frac{1}{z^{\alpha}+\lambda_{j}}\right)^{2} \leq \sum_{j=1}^{\infty}\left(\frac{1}{|z|^{\alpha}+\lambda_{j}}\right)^{2} \\
& \leq \sum_{j=1}^{\left[|z|^{s}\right]}\left(\frac{1}{|z|^{\alpha}+\lambda_{j}}\right)^{2}+\sum_{j=\left[|z|^{s}\right]+1}^{\infty}\left(\frac{1}{|z|^{\alpha}+\lambda_{j}}\right)^{2} \\
& =I_{1}+I_{2},
\end{aligned}
$$

where $[a]$ denotes the integer part of $a>1$ and we may split the summation $\sum$ into two parts since $|z|^{s}>1$ in this case.

For $I_{2}$, we have, noting that $\lambda_{j} \approx j^{2 / d}, d=1,2,3$,

$$
\begin{aligned}
I_{2} & \leq C \sum_{j=\left[|z|^{s}\right]+1}^{\infty}\left(\frac{1}{|z|^{\alpha}+j^{2 / d}}\right)^{2} \leq C \int_{|z|^{s}}^{\infty} \frac{1}{\left(|z|^{\alpha}+x^{2 / d}\right)^{2}} d x \\
& \leq \int_{1}^{\infty} \frac{1}{\left(|z|^{s} y\right)^{4 / d}}|z|^{s} d y=|z|^{s-s 4 / d} \int_{1}^{\infty} \frac{1}{y^{4 / d}} d y \leq C|z|^{s-s 4 / d}
\end{aligned}
$$

For $I_{1}$, we have

$$
\begin{aligned}
I_{1} & =\sum_{j=1}^{\left[|z|^{s}\right]}\left(\frac{1}{|z|^{\alpha}+j^{2 / d}}\right)^{2}=\sum_{j=1}^{\left[|z|^{s}\right]}\left(\frac{|z|^{\alpha}}{|z|^{\alpha}+j^{2 / d}}\right)^{2}|z|^{-2 \alpha} \\
& \leq C \sum_{j=1}^{\left[|z|^{s}\right]}|z|^{-2 \alpha} \leq C|z|^{-2 \alpha}|z|^{s}=C|z|^{s-2 \alpha} .
\end{aligned}
$$

Choosing $s>0$ such that $s-s 4 / d=s-2 \alpha$, we have for $|z|>1$, by (22) and (23),

$$
\left\|\left(z^{\alpha}+A\right)^{-1}\right\|_{H S}^{2} \leq C|z|^{-2 \alpha}|z|^{\alpha d / 2} .
$$

We now turn to the case $0<|z| \leq 1$, we have, noting that $-2 \alpha+\alpha d / 2<0$ for $d<4$,

$$
\left\|\left(z^{\alpha}+A\right)^{-1}\right\|_{H S}^{2} \leq C \sum_{j=1}^{\infty}\left(\frac{1}{|z|^{\alpha}+j^{2 / d}}\right)^{2} \leq C \sum_{j=1}^{\infty} j^{-4 / d} \leq C|z|^{0} \leq C|z|^{-2 \alpha+\alpha d / 2} .
$$

Together these estimates complete the proof of Lemma 2.4 . 
We also need the following the Lemma for the approximations to $z \in \Gamma_{\tau}$ by $z_{1}$ and $z_{2}$ defined in (10).

Lemma 2.5. Let $z_{1}$ and $z_{2}$ be defined by (10). Then we have

$$
\begin{aligned}
& \left|z_{1}\right| \sim|z|, \quad \forall z \in \Gamma_{\tau}, \\
& \left|z_{1}-z\right| \leq C \tau^{2-\alpha}|z|^{3-\alpha}, \quad \forall z \in \Gamma_{\tau}, \\
& \left|z_{2}\right| \sim|z|, \quad \forall z \in \Gamma_{\tau}, \\
& \left|z_{2}-z\right| \leq C \tau|z|^{2}, \quad \forall z \in \Gamma_{\tau},
\end{aligned}
$$

where $\left|z_{1}\right| \sim|z|, \forall z \in \Gamma_{\tau}$ means that $\left|z_{1}\right|$ and $|z|$ are equivalent on $\Gamma_{\tau}$, that is, there exist positive constants $c_{1}$ and $c_{2}$ such that $c_{1}\left|z_{1}\right| \leq|z| \leq c_{2}\left|z_{1}\right|, \forall z \in \Gamma_{\tau}$.

Proof: (24) and (25) are proved in Yan et al. [36, (3.26), (3.27)] and (26) and (27) are proved in Gunzburger et al. $[14,(3.9),(3.10)]$.

Lemma 2.6. Let $z_{1}$ and $z_{2}$ be defined by (10). Then we have

$$
\left\|\left(z^{\alpha}+A\right)^{-1} z^{-\gamma}-\left(z_{1}^{\alpha}+A\right)^{-1} z_{2}^{-\gamma} \frac{z \tau}{e^{z \tau}-1}\right\|_{H S} \leq C \tau|z|^{-\alpha-\gamma+1}\left(1+(\tau|z|)^{1-\alpha}\right)|z|^{\alpha d / 4}, \quad z \in \Gamma_{\tau} .
$$

Proof: We have

$$
\begin{aligned}
& \left\|\left(z^{\alpha}+A\right)^{-1} z^{-\gamma}-\left(z_{1}^{\alpha}+A\right)^{-1} z_{2}^{-\gamma} \frac{z \tau}{e^{z \tau}-1}\right\|_{H S} \\
\leq & \left\|\left(z^{\alpha}+A\right)^{-1} z^{-\gamma}-\left(z_{1}^{\alpha}+A\right)^{-1} z_{1}^{-\gamma}\right\|_{H S} \\
& +\left\|\left(z_{1}^{\alpha}+A\right)^{-1} z_{1}^{-\gamma}-\left(z_{1}^{\alpha}+A\right)^{-1} z_{2}^{-\gamma}\right\|_{H S} \\
& +\left\|\left(z_{1}^{\alpha}+A\right)^{-1} z_{2}^{-\gamma}-\left(z_{1}^{\alpha}+A\right)^{-1} z_{2}^{-\gamma} \frac{z \tau}{e^{z \tau}-1}\right\|_{H S} \\
= & I+I I+I I I .
\end{aligned}
$$

For $I$, we have, by mean value theorem, with $\bar{z}$ lying between $z$ and $z_{1}$,

$$
\begin{aligned}
& \left(z^{\alpha}+A\right)^{-1} z^{-\gamma}-\left(z_{1}^{\alpha}+A\right)^{-1} z_{1}^{-\gamma} \\
= & \left((-1)\left(\bar{z}^{\alpha}+A\right)^{-2} \alpha \bar{z}^{\alpha-1} \bar{z}^{-\gamma}+\left(\bar{z}^{\alpha}+A\right)^{-1}(-\gamma) \bar{z}^{-\gamma-1}\right)\left(z-z_{1}\right) .
\end{aligned}
$$

Thus we get, by Lemmas $2.4,2.5$, and noting that $\bar{z}$ and $z$ are equivalent on $\Gamma_{\tau}$, that is, $|\bar{z}| \sim z$ for $z \in \Gamma_{\tau}$,

$$
\begin{aligned}
I & =\left\|\left(z^{\alpha}+A\right)^{-1} z^{-\gamma}-\left(z_{1}^{\alpha}+A\right)^{-1} z_{1}^{-\gamma}\right\|_{H S} \\
& \leq\left\|\left((-1)\left(\bar{z}^{\alpha}+A\right)^{-2} \alpha \bar{z}^{\alpha-1} \bar{z}^{-\gamma}+\left(\bar{z}^{\alpha}+A\right)^{-1}(-\gamma) \bar{z}^{-\gamma-1}\right)\right\|_{H S}\left|z-z_{1}\right| \\
& \left.\leq C \| z^{\alpha}+A\right)^{-1} \|_{H S}|z|^{-\gamma-1}\left|z-z_{1}\right| \leq C|z|^{-\alpha-\gamma-1}|z|^{\alpha d / 4}\left|z-z_{1}\right| \\
& \leq C \tau|z|^{-\alpha-\gamma+1}(\tau|z|)^{1-\alpha}|z|^{\alpha d / 4} .
\end{aligned}
$$

We now estimate $I I$. By mean value theorem, we have, with $\bar{z}$ lying between $z$ and $z_{1}$,

$$
z_{1}^{-\gamma}-z_{2}^{-\gamma}=-\gamma \bar{z}^{-\gamma-1}\left(z_{1}-z_{2}\right)
$$

Thus we obtain, by the Lemmas 2.4, 2.5,

$$
\begin{aligned}
I I & =\left\|\left(z_{1}^{\alpha}+A\right)^{-1} z_{1}^{-\gamma}-\left(z_{1}^{\alpha}+A\right)^{-1} z_{2}^{-\gamma}\right\|_{H S} \leq\left\|\left(z_{1}^{\alpha}+A\right)^{-1}\right\|_{H S}\left|z_{1}^{-\gamma}-z_{2}^{-\gamma}\right| \\
& \leq C\left\|\left(z_{1}^{\alpha}+A\right)^{-1}\right\|_{H S}|z|^{-\gamma-1}\left|z_{1}-z_{2}\right| \leq C\left\|\left(z_{1}^{\alpha}+A\right)^{-1}\right\|_{H S}|z|^{-\gamma-1}\left(\left|z_{1}-z\right|+\left|z_{2}-z\right|\right) \\
& \leq C \tau|z|^{-\alpha-\gamma+1}\left(1+(\tau|z|)^{1-\alpha}\right)|z|^{\alpha d / 4} .
\end{aligned}
$$


Finally we have, by Lemmas $2.4,2.5$ and noting that $\left|1-\frac{z \tau}{e^{z \tau}-1}\right| \leq C|\tau z|$ for $z \in \Gamma_{\tau}$,

$$
\begin{aligned}
I I I & =\left\|\left(z_{1}^{\alpha}+A\right)^{-1} z_{2}^{-\gamma}-\left(z_{1}^{\alpha}+A\right)^{-1} z_{2}^{-\gamma} \frac{z \tau}{e^{z \tau}-1}\right\|_{H S}=\left\|\left(z_{1}^{\alpha}+A\right)^{-1} z_{2}^{-\gamma}\left(1-\frac{z \tau}{e^{z \tau}-1}\right)\right\|_{H S} \\
& \leq C\left\|\left(z_{1}^{\alpha}+A\right)^{-1}\right\|_{H S}\left|z_{2}\right|^{-\gamma}|\tau z| \leq C \tau|z|^{-\alpha-\gamma+1}|z|^{\alpha d / 4} .
\end{aligned}
$$

Together these estimates complete the proof of Lemma 2.6.

Lemma 2.7. Let $z_{1}$ and $z_{2}$ be defined by (10). We have

$$
\left\|\left[\left(z_{1}^{\alpha}+A\right)^{-1} z_{2}^{-\gamma}-\left(z_{1}^{\alpha}+A_{h}\right)^{-1} z_{2}^{-\gamma} P_{h}\right] e_{j}\right\| \leq C h^{2 \epsilon}\left(|z|^{\alpha}+\lambda_{j}\right)^{-(1-\epsilon)}|z|^{-\gamma},
$$

for all $\epsilon \in[0,1]$ and $j=1,2, \ldots, M$.

Proof: The proof is similar to the proof of Gunzburger et al. [14, Lemma 3.6]. We omit the proof here.

Now we introduce the following main theorem in this section.

Theorem 2.8. Let $\alpha+\gamma>1 / 2, \alpha \in(0,1), \gamma \in[0,1]$. Further assume that $0<2 \alpha+2 \gamma-1-\alpha d / 2<1, d=$ $1,2,3$. Let $u\left(t_{n}\right)$ and $u^{n}$ be the solutions of (1) and (7), respectively. We have

$$
\mathbb{E}\left\|u\left(t_{n}\right)-u^{n}\right\|^{2} \leq C \tau^{2 \alpha+2 \gamma-1-\alpha d / 2-\epsilon}, \quad \forall \epsilon>0 .
$$

Proof: By (6), the solution of (1) has the form, with $f(t)=\frac{d W(t)}{d t}$,

$$
u\left(t_{n}\right)=\int_{0}^{t_{n}} E\left(t_{n}-s\right) f(s) d s=\int_{0}^{t_{n}} E\left(t_{n}-s\right) d W(s),
$$

where

$$
E(t)=\frac{1}{2 \pi i} \int_{\Gamma} e^{z t}\left(z^{\alpha}+A\right)^{-1} z^{-\gamma} d z
$$

For fixed $n=1,2, \ldots, \mathrm{N}$, we denote

$$
\bar{f}(t):=\partial_{\tau} W(t):=\left\{\begin{array}{l}
\frac{W\left(t_{n}\right)-W\left(t_{n-1}\right)}{\tau}, \text { when } t \in\left(t_{n-1}, t_{n}\right] \\
0, \quad \text { when } t>t_{n} .
\end{array}\right.
$$

By the Lemma 2.1, the solution of (7) has the form

$$
u^{n}=\int_{0}^{t_{n}} E_{\tau}\left(t_{n}-s\right) \bar{f}(s) d s=\int_{0}^{t_{n}} E_{\tau}\left(t_{n}-s\right) \partial_{\tau} W(s) d s,
$$

where, with $z_{1}$ and $z_{2}$ defined by (10),

$$
E_{\tau}(t)=\frac{\tau}{2 \pi i} \int_{\Gamma_{\tau}} e^{z t}\left(z_{1}^{\alpha}+A\right)^{-1} z_{2}^{-\gamma} \frac{z}{e^{z \tau}-1} d z .
$$


Subtracting (32) from (30), we obtain

$$
\begin{aligned}
u\left(t_{n}\right)-u^{n}= & \int_{0}^{t_{n}} E\left(t_{n}-s\right) d W(s)-\int_{0}^{t_{n}} E_{\tau}\left(t_{n}-s\right) \partial_{\tau} W(s) d s \\
= & \int_{0}^{t_{n}}\left(\frac{1}{2 \pi i} \int_{\Gamma / \Gamma_{\tau}} e^{z\left(t_{n}-s\right)}\left(z^{\alpha}+A\right)^{-1} z^{-\gamma} d z\right) d W(s) \\
+ & \left\{\int _ { 0 } ^ { t _ { n } } \left[\frac{1}{2 \pi i} \int_{\Gamma_{\tau}} e^{z\left(t_{n}-s\right)}\left(z^{\alpha}+A\right)^{-1} z^{-\gamma} d z\right.\right. \\
& \left.\left.-\frac{\tau}{2 \pi i} \int_{\Gamma_{\tau}} e^{z\left(t_{n}-s\right)}\left(z_{1}^{\alpha}+A\right)^{-1} z_{2}^{-\gamma} \frac{z}{e^{z \tau}-1} d z\right] d W(s)\right\} \\
+ & \left\{\int_{0}^{t_{n}}\left(\frac{\tau}{2 \pi i} \int_{\Gamma_{\tau}} e^{z\left(t_{n}-s\right)}\left(z_{1}^{\alpha}+A\right)^{-1} z_{2}^{-\gamma} \frac{z}{e^{z \tau}-1} d z\right) d W(s)\right. \\
& \left.-\int_{0}^{t_{n}}\left(\frac{\tau}{2 \pi i} \int_{\Gamma_{\tau}} e^{z\left(t_{n}-s\right)}\left(z_{1}^{\alpha}+A\right)^{-1} z_{2}^{-\gamma} \frac{z}{e^{z \tau}-1} d z\right) \partial_{\tau} W(s) d s\right\} \\
=I+ & I I+I I I .
\end{aligned}
$$

For $I$, we have, by Lemmas 2.2 and 2.4 and the Cauchy-Schwarz inequality, with some suitable constant $c>0$,

$$
\begin{aligned}
\mathbb{E}\|I\|^{2} & =\mathbb{E}\left\|\int_{0}^{t_{n}}\left(\frac{1}{2 \pi i} \int_{\Gamma / \Gamma_{\tau}} e^{z\left(t_{n}-s\right)}\left(z^{\alpha}+A\right)^{-1} z^{-\gamma} d z\right) d W(s)\right\|^{2} \\
& =\int_{0}^{t_{n}}\left\|\frac{1}{2 \pi i} \int_{\Gamma / \Gamma_{\tau}} e^{z\left(t_{n}-s\right)}\left(z^{\alpha}+A\right)^{-1} z^{-\gamma} d z\right\|_{H S}^{2} d s \\
& \leq C \int_{0}^{t_{n}}\left[\int_{\Gamma / \Gamma_{\tau}}\left|e^{z\left(t_{n}-s\right)}\right|\left\|\left(z^{\alpha}+A\right)^{-1}\right\|_{H S}|z|^{-\gamma}|d z|\right]^{2} d s \\
& \leq C \int_{0}^{t_{n}}\left[\int_{\tau^{-1}}^{\infty} e^{-c r\left(t_{n}-s\right)} r^{-\alpha+\alpha d / 4} r^{-\gamma} d r\right]^{2} d s .
\end{aligned}
$$

Let $\beta>0$ be some suitable constant which we will determine later. We then have

$$
\begin{aligned}
\mathbb{E}\|I\|^{2} & \leq C \int_{0}^{t_{n}}\left[\int_{\tau^{-1}}^{\infty} e^{-c r\left(t_{n}-s\right)} r^{-\beta} r^{\beta} r^{-\alpha+\alpha d / 4} r^{-\gamma} d r\right]^{2} d s \\
& \leq C \tau^{2 \beta} \int_{0}^{t_{n}}\left[\int_{\tau^{-1}}^{\infty} e^{-c r\left(t_{n}-s\right)} r^{\beta-\alpha+\alpha d / 4-\gamma} d r\right]^{2} d s .
\end{aligned}
$$

Note that, with $\beta-\alpha+\alpha d / 4-\gamma>-1$,

$$
\begin{aligned}
\int_{\tau^{-1}}^{\infty} e^{-c r\left(t_{n}-s\right)} r^{\beta-\alpha+\alpha d / 4-\gamma} d r & \leq C\left(t_{n}-s\right)^{-\beta+\alpha-\alpha d / 4+\gamma-1}\left(\int_{0}^{\infty} e^{-c x} x^{\beta-\alpha+\alpha d / 4-\gamma} d x\right) \\
& \leq C\left(t_{n}-s\right)^{-\beta+\alpha-\alpha d / 4+\gamma-1} .
\end{aligned}
$$

Thus we have, with $2(-\beta+\alpha-\alpha d / 4+\gamma-1)>-1$,

$$
\mathbb{E}\|I\|^{2} \leq C \tau^{2 \beta} \int_{0}^{t_{n}}\left(t_{n}-s\right)^{2(-\beta+\alpha-\alpha d / 4+\gamma-1)} \leq C \tau^{2 \beta} .
$$


Now let us choose the following $\beta>0$ such that, with $\epsilon>0$,

$$
2 \beta=2(\alpha+\gamma)-1-\alpha d / 2-\epsilon .
$$

We observe that $\beta>0$ in (37) satisfies the following conditions

$$
\beta-\alpha+\alpha d / 4-\gamma=-1 / 2-\epsilon / 2>-1,
$$

and

Hence (36) implies that

$$
2(-\beta+\alpha-\alpha d / 4+\gamma-1)=-1+\epsilon>-1
$$

$$
\mathbb{E}\|I\|^{2} \leq C \tau^{2(\alpha+\gamma)-1-\alpha d / 2-\epsilon}, \forall \epsilon>0 .
$$

For $I I$, we have, by the Lemma 2.2 ,

$$
\begin{aligned}
\mathbb{E}\|I I\|^{2}= & \int_{0}^{t_{n}} \| \frac{1}{2 \pi i} \int_{\Gamma_{\tau}} e^{z\left(t_{n}-s\right)}\left(z^{\alpha}+A\right)^{-1} z^{-\gamma} d z \\
& -\frac{\tau}{2 \pi i} \int_{\Gamma_{\tau}} e^{z\left(t_{n}-s\right)}\left(z_{1}^{\alpha}+A\right)^{-1} z_{2}^{-\gamma} \frac{z}{e^{z \tau}-1} d z \|_{H S}^{2} d s .
\end{aligned}
$$

Note that, by using the Cauchy-Schwarz inequality,

$$
\begin{aligned}
& \left\|\frac{1}{2 \pi i} \int_{\Gamma_{\tau}} e^{z\left(t_{n}-s\right)}\left(z^{\alpha}+A\right)^{-1} z^{-\gamma} d z-\frac{\tau}{2 \pi i} \int_{\Gamma_{\tau}} e^{z\left(t_{n}-s\right)}\left(z_{1}^{\alpha}+A\right)^{-1} z_{2}^{-\gamma} \frac{z}{e^{z \tau}-1} d z\right\|_{H S}^{2} \\
& \leq C\left(\int_{\Gamma_{\tau}} 1^{2}|d z|\right)\left(\int_{\Gamma_{\tau}}\left|e^{z\left(t_{n}-s\right)}\right|^{2}\left\|\left(z^{\alpha}+A\right)^{-1} z^{-\gamma}-\left(z_{1}^{\alpha}+A\right)^{-1} z_{2}^{-\gamma} \frac{z}{e^{z \tau}-1}\right\|_{H S}^{2}|d z|\right) .
\end{aligned}
$$

We then have, by the Lemma 2.6, with some suitable constant $c>0$,

$$
\begin{aligned}
\mathbb{E}\|I I\|^{2} & \leq C \int_{0}^{t_{n}}\left(\int_{\Gamma_{\tau}} 1^{2}|d z|\right)\left(\int_{\Gamma_{\tau}}\left|e^{z\left(t_{n}-s\right)}\right|^{2}\left\|\left(z^{\alpha}+A\right)^{-1} z^{-\gamma}-\left(z_{1}^{\alpha}+A\right)^{-1} z_{2}^{-\gamma} \frac{z}{e^{z \tau}-1}\right\|_{H S}^{2}|d z|\right) d s \\
& =C \int_{0}^{t_{n}}\left(\int_{\Gamma_{\tau}} 1^{2}|d z|\right)\left(\int_{\Gamma_{\tau}}\left|e^{z s}\right|^{2}\left(\tau^{2}|z|^{2(-\alpha-\gamma+1)}|z|^{\alpha d / 2}\right)\left(1+|\tau z|^{1-\alpha}\right)^{2}|d z|\right) d s \\
& \leq C \int_{0}^{t_{n}}\left(\int_{0}^{\tau^{-1}} d r\right)\left(\int_{0}^{\tau^{-1}} e^{-c r s}\left(\tau^{2} r^{2(-\alpha-\gamma+1)} r^{\alpha d / 2}\right)\left(1+(\tau r)^{2(1-\alpha)}\right) d r\right) d s \\
& \leq C\left(\int_{0}^{\tau^{-1}} d r\right)\left[\int_{0}^{\tau^{-1}}\left(\int_{0}^{t_{n}} e^{-c r s} d s\right)\left(\tau^{2} r^{2(-\alpha-\gamma+1)} r^{\alpha d / 2}\right)\left(1+(\tau r)^{2(1-\alpha)}\right) d r\right] \\
& \leq C \tau^{-1} \int_{0}^{\tau^{-1}} r^{-1}\left(\tau^{2} r^{2(-\alpha-\gamma+1)} r^{\alpha d / 2}\right)\left(1+(\tau r)^{2(1-\alpha)}\right) d r .
\end{aligned}
$$
have

Using the assumption $2 \alpha+2 \gamma-1-\alpha d / 2<1$ which implies that $-1+2(-\alpha-\gamma+1)+\alpha d / 2>-1$, we

$$
\begin{aligned}
\mathbb{E}\|I I\|^{2} & \leq C \tau^{-1} \int_{0}^{\tau^{-1}} r^{-1}\left(\tau^{2} r^{2(-\alpha-\gamma+1)} r^{\alpha d / 2}\right)\left(1+(\tau r)^{2(1-\alpha)}\right) \\
& =C \tau \int_{0}^{\tau^{-1}} r^{-1+2(-\alpha-\gamma+1)+\alpha d / 2} d s+C \tau^{1+2(1+\alpha)} \int_{0}^{\tau^{-1}} r^{-1+2(-\alpha-\gamma+1)+\alpha d / 2+2(1-\alpha)} d s \\
& \leq C \tau^{2(\alpha+\gamma)-1-\alpha d / 2} .
\end{aligned}
$$


Finally we estimate $I I I$. We have

$$
\begin{aligned}
\mathbb{E}\|I I I\|^{2}= & \mathbb{E} \| \int_{0}^{t_{n}}\left(\frac{\tau}{2 \pi i} \int_{\Gamma_{\tau}} e^{z\left(t_{n}-s\right)}\left(z_{1}^{\alpha}+A\right)^{-1} z_{2}^{-\gamma} \frac{z}{e^{z \tau}-1} d z\right) d W(s) \\
& -\sum_{i=1}^{n} \int_{t_{i-1}}^{t_{i}}\left(\frac{\tau}{2 \pi i} \int_{\Gamma_{\tau}} e^{z\left(t_{n}-\bar{s}\right)}\left(z_{1}^{\alpha}+A\right)^{-1} z_{2}^{-\gamma} \frac{z}{e^{z \tau}-1} d z\right)\left(\frac{1}{\tau} \int_{t_{i-1}}^{t_{i}} d W(s)\right) d \bar{s} \|^{2} \\
= & \mathbb{E} \| \sum_{i=1}^{n} \int_{t_{i-1}}^{t_{i}}\left[\frac{1}{\tau} \int_{t_{i-1}}^{t_{i}}\left(\frac{\tau}{2 \pi i} \int_{\Gamma_{\tau}} e^{z\left(t_{n}-s\right)}\left(z_{1}^{\alpha}+A\right)^{-1} z_{2}^{-\gamma} \frac{z}{e^{z \tau}-1} d z\right) d \bar{s}\right] d W(s) \\
& -\sum_{i=1}^{n} \int_{t_{i-1}}^{t_{i}}\left[\frac{1}{\tau} \int_{t_{i-1}}^{t_{i}}\left(\frac{\tau}{2 \pi i} \int_{\Gamma_{\tau}} e^{z\left(t_{n}-\bar{s}\right)}\left(z_{1}^{\alpha}+A\right)^{-1} z_{2}^{-\gamma} \frac{z}{e^{z \tau}-1} d z\right) d \bar{s}\right] d W(s) \|^{2} .
\end{aligned}
$$

By using the isometry property Lemma 2.2 and the Cauchy-Schwarz inequality, we have

$$
\begin{aligned}
\mathbb{E}\|I I I\|^{2}= & \sum_{i=1}^{n} \int_{t_{i-1}}^{t_{i}} \|\left\{\left[\frac{1}{\tau} \int_{t_{i-1}}^{t_{i}}\left(\frac{\tau}{2 \pi i} \int_{\Gamma_{\tau}} e^{z\left(t_{n}-s\right)}\left(z_{1}^{\alpha}+A\right)^{-1} z_{2}^{-\gamma} \frac{z}{e^{z \tau}-1} d z\right) d \bar{s}\right]\right. \\
& \left.-\left[\frac{1}{\tau} \int_{t_{i-1}}^{t_{i}}\left(\frac{\tau}{2 \pi i} \int_{\Gamma_{\tau}} e^{z\left(t_{n}-\bar{s}\right)}\left(z_{1}^{\alpha}+A\right)^{-1} z_{2}^{-\gamma} \frac{z}{e^{z \tau}-1} d z\right) d \bar{s}\right]\right\} \|_{H S}^{2} d s \\
\leq & C \sum_{i=1}^{n} \int_{t_{i-1}}^{t_{i}}\left\{\frac{1}{\tau^{2}}\left(\int_{t_{i-1}}^{t_{i}} 1^{2} d \bar{s}\right) \int_{t_{i-1}}^{t_{i}} \|\left(\frac{\tau}{2 \pi i} \int_{\Gamma_{\tau}} e^{z\left(t_{n}-s\right)}\left(z_{1}^{\alpha}+A\right)^{-1} z_{2}^{-\gamma} \frac{z}{e^{z \tau}-1} d z\right)\right. \\
& \left.-\left(\frac{\tau}{2 \pi i} \int_{\Gamma_{\tau}} e^{z\left(t_{n}-\bar{s}\right)}\left(z_{1}^{\alpha}+A\right)^{-1} z_{2}^{-\gamma} \frac{z}{e^{z \tau}-1} d z\right) \|_{H S}^{2} d \bar{s}\right\} d s \\
= & C \frac{1}{\tau} \sum_{i=1}^{n} \int_{t_{i-1}}^{t_{i}}\left\{\int_{t_{i-1}}^{t_{i}} \|\left(\frac{\tau}{2 \pi i} \int_{\Gamma_{\tau}} e^{z\left(t_{n}-s\right)}\left(z_{1}^{\alpha}+A\right)^{-1} z_{2}^{-\gamma} \frac{z}{e^{z \tau}-1} d z\right)\right. \\
& \left.-\left(\frac{\tau}{2 \pi i} \int_{\Gamma_{\tau}} e^{z\left(t_{n}-\bar{s}\right)}\left(z_{1}^{\alpha}+A\right)^{-1} z_{2}^{-\gamma} \frac{z}{e^{z \tau}-1} d z\right) \|_{H S}^{2} d \bar{s}\right\} d s .
\end{aligned}
$$

Note that, by the Cauchy-Schwarz inequality,

$$
\begin{aligned}
& \left\|\left(\frac{\tau}{2 \pi i} \int_{\Gamma_{\tau}} e^{z\left(t_{n}-s\right)}\left(z_{1}^{\alpha}+A\right)^{-1} z_{2}^{-\gamma} \frac{z}{e^{z \tau}-1} d z\right)-\left(\frac{\tau}{2 \pi i} \int_{\Gamma_{\tau}} e^{z\left(t_{n}-\bar{s}\right)}\left(z_{1}^{\alpha}+A\right)^{-1} z_{2}^{-\gamma} \frac{z}{e^{z \tau}-1} d z\right)\right\|_{H S}^{2} \\
& =\frac{1}{2 \pi}\left\|\int_{\Gamma_{\tau}} e^{z\left(t_{n}-s\right)}\left(1-e^{z(s-\bar{s})}\right)\left(z_{1}^{\alpha}+A\right)^{-1} z_{2}^{-\gamma} \frac{z \tau}{e^{z \tau}-1} d z\right\|_{H S}^{2} \\
& \leq C\left(\int_{\Gamma_{\tau}}|d z|\right)\left[\int_{\Gamma_{\tau}}\left|e^{z\left(t_{n}-s\right)}\right|^{2}\left(\left|1-e^{z(s-\bar{s})}\right|^{2}\right)\left\|\left(z_{1}^{\alpha}+A\right)^{-1} z_{2}^{-\gamma}\right\|_{H S}^{2}\left|\frac{z \tau}{e^{z \tau}-1}\right|^{2}|d z|\right] .
\end{aligned}
$$

It is easy to show that, with $s, \bar{s} \in\left(t_{i-1}, t_{i}\right)$, see, Yan et al. [36],

$$
\left|1-e^{z(s-\bar{s})}\right| \leq C|z| \tau, \quad\left|\frac{z \tau}{e^{z \tau}-1}\right| \leq C, \quad \forall z \in \Gamma_{\tau} .
$$

Combining (40) with (41), we have

$$
\begin{aligned}
& \left\|\left(\frac{\tau}{2 \pi i} \int_{\Gamma_{\tau}} e^{z\left(t_{n}-s\right)}\left(z_{1}^{\alpha}+A\right)^{-1} z_{2}^{-\gamma} \frac{z}{e^{z \tau}-1} d z\right)-\left(\frac{\tau}{2 \pi i} \int_{\Gamma_{\tau}} e^{z\left(t_{n}-\bar{s}\right)}\left(z_{1}^{\alpha}+A\right)^{-1} z_{2}^{-\gamma} \frac{z}{e^{z \tau}-1} d z\right)\right\|_{H S}^{2} \\
& \leq C\left(\int_{\Gamma_{\tau}}|d z|\right)\left[\int_{\Gamma_{\tau}}\left|e^{z\left(t_{n}-s\right)}\right|^{2}|z|^{2} \tau^{2}\left\|\left(z_{1}^{\alpha}+A\right)^{-1} z_{2}^{-\gamma}\right\|_{H S}^{2}|d z|\right] \\
& \leq C\left(\int_{\Gamma_{\tau}}|d z|\right)\left[\int_{\Gamma_{\tau}}\left|e^{z\left(t_{n}-s\right)}\right|^{2}|z|^{2} \tau^{2}\left\|\left(z_{1}^{\alpha}+A\right)^{-1} z_{2}^{-\gamma}\right\|_{H S}^{2}|d z|\right] .
\end{aligned}
$$


Hence

$$
\begin{aligned}
\mathbb{E}\|I I I\|^{2} & \leq C \frac{1}{\tau} \sum_{i=1}^{n} \int_{t_{i-1}}^{t_{i}}\left\{\int_{t_{i-1}}^{t_{i}}\left(\int_{0}^{\tau^{-1}} d r\right)\left[\int_{\Gamma_{\tau}}\left|e^{z\left(t_{n}-s\right)}\right|^{2}|z|^{2} \tau^{2}\left\|\left(z_{1}^{\alpha}+A\right)^{-1} z_{2}^{-\gamma}\right\|_{H S}^{2}|d z|\right] d \bar{s}\right\} d s \\
& =C \frac{1}{\tau} \sum_{i=1}^{n} \int_{t_{i-1}}^{t_{i}}\left\{\int_{t_{i-1}}^{t_{i}} \tau\left[\int_{\Gamma_{\tau}}\left|e^{z\left(t_{n}-s\right)}\right|^{2}|z|^{2}\left\|\left(z_{1}^{\alpha}+A\right)^{-1} z_{2}^{-\gamma}\right\|_{H S}^{2}|d z|\right] d \bar{s}\right\} d s \\
& \leq C \tau \int_{0}^{t_{n}}\left[\int_{\Gamma_{\tau}}\left|e^{z\left(t_{n}-s\right)}\right|^{2}|z|^{2}\left\|\left(z_{1}^{\alpha}+A\right)^{-1} z_{2}^{-\gamma}\right\|_{H S}^{2}|d z|\right] d s \\
& \leq C \tau \int_{0}^{t_{n}}\left[\int_{\Gamma_{\tau}}\left|e^{z s}\right|^{2}|z|^{2}\left\|\left(z_{1}^{\alpha}+A\right)^{-1} z_{2}^{-\gamma}\right\|_{H S}^{2}|d z|\right] d s
\end{aligned}
$$

By Lemmas 2.4 and 2.5, we have, with some suitable constant $c>0$,

$$
\begin{aligned}
\mathbb{E}\|I I I\|^{2} & \leq C \tau \int_{0}^{t_{n}}\left[\int_{0}^{\tau^{-1}} e^{-c r s} r^{2-2 \alpha} r^{\alpha d / 2-2 \gamma} d r\right] d s \leq C \tau \int_{0}^{\tau^{-1}}\left(\int_{0}^{t_{n}} e^{-c r s} d s\right) r^{\alpha d / 2+2-2(\alpha+\gamma)} d r \\
& \leq C \tau \int_{0}^{\tau^{-1}} r^{-1} r^{\alpha d / 2+2-2(\alpha+\gamma)} d r \leq C \tau^{2(\alpha+\gamma)-1-\alpha d / 2}
\end{aligned}
$$

where the last inequality is estimated by using the same idea as in (39).

Together these estimates complete the proof of Theorem 2.8 .

Remark 2.1. From Theorem 2.8, we have the following error estimates in some special cases:

1. When $\alpha=1, \gamma=0$, the time convergence rate is $O\left(\tau^{1 / 4-\epsilon}\right), \epsilon>0$ in one-dimensional case, which is consistent with the results in Yan et al. [36].

2. When $\alpha+\gamma=1$, the time convergence rate is $O\left(\tau^{1-\alpha d / 2-\epsilon}\right) \epsilon>0$ which is consistent with the results in Gunzburger et al. [15].

\section{Space discretization}

In this section, we shall consider the space discretization of (7). Let $\mathcal{T}_{h}$ be the triangulation on $\mathcal{D} \subset$ $\mathbb{R}^{d}, d=1,2,3$ and $h$ the space step size. Let $S_{h}$ be the linear finite element space defined on $\mathcal{T}_{h}$. The finite element method of $(7)$ is to find $u_{h}^{n}, n=1,2, \ldots, N$ such that

$$
\tau^{-\alpha} \sum_{k=0}^{n} w_{n-k} u_{h}^{k}+A_{h} u_{h}^{n}=P_{h} f_{h}^{n}, n=1,2, \ldots, N, \quad u_{h}^{0}=0,
$$

where

$$
f_{h}^{n}:=\sum_{l=1}^{M} e_{l} \frac{\beta_{l}\left(t_{n}\right)-\beta_{l}\left(t_{n-1}\right)}{\tau},
$$

Here $M \sim h^{-d}$ and $P_{h}: L^{2}(\mathcal{D}) \rightarrow S_{h}$ denotes the $L^{2}$-projection defined by

$$
\left(P_{h} v, \chi\right)=(v, \chi), \forall \chi \in S_{h},
$$

and $A_{h}: S_{h} \rightarrow S_{h}$ denotes the discrete Laplacian defined by

$$
\left(A_{h} \psi, \chi\right)=A(\psi, \chi), \forall \psi, \chi \in S_{h},
$$

where $A(\psi, \chi)$ is the bilinear form corresponding to the elliptic operator $A$ defined on $S_{h} \times S_{h}$.

We now introduce the following main theorem in this section. 
Theorem 3.1. Let $\alpha+\gamma>1 / 2, \alpha \in(0,1), \gamma \in[0,1]$. Assume that $0 \leq \epsilon<\frac{2(\alpha+\gamma)-1}{2 \alpha}$. Let $u^{n}$ and $u_{h}^{n}$ be the solutions of (7) and (44), respectively. Then we have

$$
\mathbb{E}\left\|u^{n}-u_{h}^{n}\right\|^{2} \leq C h^{4 \epsilon-d} \tau^{-2 \epsilon \alpha+2(\alpha+\gamma)-1}+C \tau^{2(\alpha+\gamma)-1-\alpha d / 2} .
$$

Proof: By the Lemma 2.1, the solution of (7) has the form, with $z_{1}$ and $z_{2}$ defined by (10),

$$
u^{n}=\sum_{j=1}^{\infty} \sum_{l=1}^{n} \int_{t_{l-1}}^{t_{l}} E_{\tau}\left(t_{n}-s\right) e_{j} \frac{\beta_{j}\left(t_{l}\right)-\beta_{j}\left(t_{l-1}\right)}{\tau} d s
$$

where

$$
E_{\tau}(t)=\frac{\tau}{2 \pi i} \int_{\Gamma_{\tau}} e^{z t}\left(z_{1}^{\alpha}+A\right)^{-1} z_{2}^{-\gamma} \frac{z}{e^{z \tau}-1} d z .
$$

Similarly, the solution of (44) has the following form,

$$
u_{h}^{n}=\sum_{j=1}^{M} \sum_{l=1}^{n} \int_{t_{l-1}}^{t_{l}} E_{\tau}^{h}\left(t_{n}-s\right) e_{j} \frac{\beta_{j}\left(t_{l}\right)-\beta_{j}\left(t_{l-1}\right)}{\tau} d s,
$$

where

Hence we have

$$
E_{\tau}^{h}(t)=\frac{\tau}{2 \pi i} \int_{\Gamma_{\tau}} e^{z t}\left(z_{1}^{\alpha}+A_{h}\right)^{-1} z_{2}^{-\gamma} \frac{z}{e^{z \tau}-1} P_{h} d z
$$

$$
\begin{aligned}
u^{n}-u_{h}^{n}= & \sum_{j=1}^{M} \sum_{l=1}^{n} \int_{t_{l-1}}^{t_{l}}\left[E_{\tau}\left(t_{n}-s\right)-E_{\tau}^{h}\left(t_{n}-s\right)\right] e_{j} \frac{\beta_{j}\left(t_{l}\right)-\beta_{j}\left(t_{l-1}\right)}{\tau} d s \\
& +\sum_{j=M+1}^{\infty} \sum_{l=1}^{n} \int_{t_{l-1}}^{t_{l}} E_{\tau}\left(t_{n}-s\right) e_{j} \frac{\beta_{j}\left(t_{l}\right)-\beta_{j}\left(t_{l-1}\right)}{\tau} d s \\
= & I+I I .
\end{aligned}
$$

For $I$, we have, by the Lemma 2.2,

$$
\begin{aligned}
\mathbb{E}\|I\|^{2} & =\mathbb{E}\left\|\sum_{j=1}^{M} \sum_{l=1}^{n} \int_{t_{l-1}}^{t_{l}}\left[E_{\tau}\left(t_{n}-s\right)-E_{\tau}^{h}\left(t_{n}-s\right)\right] e_{j} \frac{\beta_{j}\left(t_{l}\right)-\beta_{j}\left(t_{l-1}\right)}{\tau} d s\right\|^{2} \\
& =\mathbb{E}\left\|\sum_{j=1}^{M} \frac{1}{\tau} \sum_{l=1}^{n} \int_{t_{l-1}}^{t_{l}}\left\{\int_{t_{l-1}}^{t_{l}}\left[E_{\tau}\left(t_{n}-\bar{s}\right)-E_{\tau}^{h}\left(t_{n}-\bar{s}\right)\right] e_{j} d \bar{s}\right\} d \beta_{j}(s)\right\|^{2} \\
& =\sum_{j=1}^{M} \frac{1}{\tau^{2}} \sum_{l=1}^{n} \int_{t_{l-1}}^{t_{l}}\left\|\int_{t_{l-1}}^{t_{l}}\left[E_{\tau}\left(t_{n}-\bar{s}\right)-E_{\tau}^{h}\left(t_{n}-\bar{s}\right)\right] e_{j} d \bar{s}\right\|^{2} d s \\
& =\sum_{j=1}^{M} \frac{1}{\tau} \sum_{l=1}^{n}\left\|\int_{t_{l-1}}^{t_{l}}\left[E_{\tau}\left(t_{n}-\bar{s}\right)-E_{\tau}^{h}\left(t_{n}-\bar{s}\right)\right] e_{j} d \bar{s}\right\|^{2} .
\end{aligned}
$$

By using the Cauchy-Schwarz inequality, we have

$$
\begin{aligned}
\mathbb{E}\|I\|^{2} & \leq \sum_{j=1}^{M} \frac{1}{\tau} \sum_{l=1}^{n}\left[\int_{t_{l-1}}^{t_{l}} 1^{2} d \bar{s}\right]\left[\int_{t_{l-1}}^{t_{l}}\left\|\left(E_{\tau}\left(t_{n}-\bar{s}\right)-E_{\tau}^{h}\left(t_{n}-\bar{s}\right)\right) e_{j}\right\|^{2} d \bar{s}\right] \\
& =\sum_{j=1}^{M} \sum_{l=1}^{n} \int_{t_{l-1}}^{t_{l}}\left\|\left(E_{\tau}\left(t_{n}-\bar{s}\right)-E_{\tau}^{h}\left(t_{n}-\bar{s}\right)\right) e_{j}\right\|^{2} d \bar{s}=\sum_{j=1}^{M} \int_{0}^{t_{n}}\left\|\left(E_{\tau}(t)-E_{\tau}^{h}(t)\right) e_{j}\right\|^{2} d t .
\end{aligned}
$$


Note that

$$
\begin{aligned}
\left\|\left(E_{\tau}(t)-E_{\tau}^{h}(t)\right) e_{j}\right\|^{2} & =\left\|\frac{1}{2 \pi i} \int_{\Gamma_{\tau}} e^{z t} \frac{z \tau}{e^{z \tau}-1}\left[\left(z_{1}^{\alpha}+A\right)^{-1} z_{2}^{-\gamma}-\left(z_{1}^{\alpha}+A_{h}\right)^{-1} z_{2}^{-\gamma} P_{h}\right] e_{j} d z\right\|^{2} \\
& \leq C\left[\int_{\Gamma_{\tau}}\left|e^{z t}\right|\left|\frac{z \tau}{e^{z \tau}-1}\right|\left\|\left[\left(z_{1}^{\alpha}+A\right)^{-1} z_{2}^{-\gamma}-\left(z_{1}^{\alpha}+A_{h}\right)^{-1} z_{2}^{-\gamma} P_{h}\right] e_{j}\right\||d z|\right]^{2}
\end{aligned}
$$

By the Lemma 2.7, we have, with $\epsilon \in[0,1]$ and some suitable constants $\beta \in(0,1)$ and $c>0$,

$$
\begin{aligned}
\left\|\left(E_{\tau}(t)-E_{\tau}^{h}(t)\right) e_{j}\right\|^{2} & \leq C\left[\int_{\Gamma_{\tau}}\left|e^{z t}\right| h^{2 \epsilon}\left(|z|^{\alpha}+\lambda_{j}\right)^{-(1-\epsilon)}|z|^{-\gamma}|d z|\right]^{2} \\
& \leq C\left[\int_{0}^{\tau^{-1}}\left|e^{-c r t}\right| h^{2 \epsilon}\left(r^{\alpha}+\lambda_{j}\right)^{-(1-\epsilon)} r^{-\gamma} d r\right]^{2} \\
& \leq C h^{4 \epsilon}\left[\int_{0}^{\tau^{-1}}\left(e^{-c r t / 2} r^{-\beta / 2}\right)\left(e^{-c r t / 2} r^{\beta / 2}\left(r^{\alpha}+\lambda_{j}\right)^{-(1-\epsilon)}\right) r^{-\gamma} d r\right]^{2} \\
& \leq C h^{4 \epsilon} \int_{0}^{\tau^{-1}} e^{-c r t} r^{-\beta} d r \int_{0}^{\tau^{-1}}\left(e^{-c r t} r^{\beta}\left(r^{\alpha}+\lambda_{j}\right)^{-(2-2 \epsilon)}\right) r^{-2 \gamma} d r .
\end{aligned}
$$

Note that $\int_{0}^{\tau^{-1}} e^{-c r t} r^{-\beta} d r \leq t^{\beta-1} \int_{0}^{\infty} e^{-c x} x^{-\beta} d x \leq C t^{\beta-1}$ for $\beta \in(0,1), c>0$, we obtain

$$
\left\|\left(E_{\tau}(t)-E_{\tau}^{h}(t)\right) e_{j}\right\|^{2} \leq C h^{4 \epsilon} t^{\beta-1}\left[\int_{0}^{\tau^{-1}} e^{-c r t} \frac{r^{\beta-2 \gamma}}{\left(r^{\alpha}+\lambda_{j}\right)^{2-2 \epsilon}} d r\right] .
$$

Hence we have

$$
\begin{aligned}
\mathbb{E}\|I\|^{2} & \leq C \sum_{j=1}^{M} \int_{0}^{t_{n}} C h^{4 \epsilon} t^{\beta-1}\left[\int_{0}^{\tau^{-1}} e^{-c r t} \frac{r^{\beta-2 \gamma}}{\left(r^{\alpha}+\lambda_{j}\right)^{2-2 \epsilon}} d r\right] d t \\
& \leq C h^{4 \epsilon} \int_{0}^{t_{n}} t^{\beta-1}\left[\int_{0}^{\tau^{-1}} e^{-c r t} \sum_{j=1}^{M} \frac{r^{\beta-2 \gamma}}{\left(r^{\alpha}+\lambda_{j}\right)^{2-2 \epsilon}} d r\right] d t \\
& =h^{4 \epsilon} \int_{0}^{t_{n}} t^{\beta-1}\left[\int_{0}^{\tau^{-1}} e^{-c r t} r^{2 \epsilon \alpha-2(\alpha+\gamma)+\beta} \sum_{j=1}^{M}\left(\frac{r^{\alpha}}{r^{\alpha}+\lambda_{j}}\right)^{2-2 \epsilon} d r\right] d t
\end{aligned}
$$

Note that $\sum_{j=1}^{M}\left(\frac{r^{\alpha}}{r^{\alpha}+\lambda_{j}}\right)^{2-2 \epsilon} \leq \sum_{j=1}^{M} 1=M \leq C h^{-d}$, we have, if $\alpha+\gamma>1 / 2, \alpha \in(0,1), \gamma \in[0,1]$ and $-2 \epsilon \alpha+2(\alpha+\gamma)-1>0$, i.e., $0 \leq \epsilon<\frac{2(\alpha+\gamma)-1}{2 \alpha}$,

$$
\begin{aligned}
\mathbb{E}\|I\|^{2} & \leq C h^{4 \epsilon-d} \int_{0}^{t_{n}} t^{\beta-1}\left[\int_{0}^{\tau^{-1}} e^{-c r t} r^{2 \epsilon \alpha-2(\alpha+\gamma)+\beta} d r\right] d t \\
& \leq C h^{4 \epsilon-d} \int_{0}^{\tau^{-1}}\left[\int_{0}^{t_{n}} t^{\beta-1} e^{-c r t} d t\right] r^{2 \epsilon \alpha-2(\alpha+\gamma)+\beta} d r \\
& \leq C h^{4 \epsilon-d} \int_{0}^{\tau^{-1}} r^{-\beta} r^{2 \epsilon \alpha-2(\alpha+\gamma)+\beta} d r \leq C h^{4 \epsilon-d} \tau^{-2 \epsilon \alpha+2(\alpha+\gamma)-1} .
\end{aligned}
$$


For $I I$, we have, by the Lemma 2.2 and the Cauchy-Schwarz inequality,

$$
\begin{aligned}
\mathbb{E}\|I I\|^{2} & =\mathbb{E}\left\|\sum_{j=M+1}^{\infty} \sum_{l=1}^{n} \int_{t_{l-1}}^{t_{l}} E_{\tau}\left(t_{n}-s\right) e_{j} \frac{\beta_{j}\left(t_{l}\right)-\beta_{j}\left(t_{l-1}\right)}{\tau} d s\right\|^{2} \\
& =\mathbb{E}\left\|\sum_{j=M+1}^{\infty} \frac{1}{\tau} \int_{t_{l-1}}^{t_{l}}\left[\sum_{l=1}^{n} \int_{t_{l-1}}^{t_{l}} E_{\tau}\left(t_{n}-\bar{s}\right) e_{j} d \bar{s}\right] d \beta_{j}(s)\right\|^{2} \\
& =\sum_{j=M+1}^{\infty} \frac{1}{\tau^{2}} \int_{t_{l-1}}^{t_{l}} \sum_{l=1}^{n}\left\|\int_{t_{l-1}}^{t_{l}} E_{\tau}\left(t_{n}-\bar{s}\right) e_{j} d \bar{s}\right\|^{2} d s \\
& =\sum_{j=M+1}^{\infty} \frac{1}{\tau} \sum_{l=1}^{n}\left\|\int_{t_{l-1}}^{t_{l}} E_{\tau}\left(t_{n}-\bar{s}\right) e_{j} d \bar{s}\right\|^{2} \\
& \leq C \sum_{j=M+1}^{\infty} \frac{1}{\tau} \sum_{l=1}^{n}\left(\int_{t_{l-1}}^{t_{l}} 1^{2} d \bar{s}\right)\left(\int_{t_{l-1}}^{t_{l}}\left\|E_{\tau}\left(t_{n}-\bar{s}\right) e_{j}\right\|^{2} d \bar{s}\right) \\
& \leq C \sum_{j=M+1}^{\infty} \int_{0}^{t_{n}}\left\|E_{\tau}(t) e_{j}\right\|^{2} d t=C \int_{0}^{t_{n}} \sum_{j=M+1}^{\infty}\left\|E_{\tau}(t) e_{j}\right\|^{2} d t .
\end{aligned}
$$

Note that

$$
\begin{aligned}
\left\|E_{\tau}(t) e_{j}\right\|^{2} & =\left\|\mid \frac{1}{2 \pi i} \int_{\Gamma_{\tau}} e^{z t}\left(z_{1}^{\alpha}+\lambda_{j}\right)^{-1} z_{2}^{-\gamma} \frac{z \tau}{e^{z \tau}-1} e_{j} d z\right\|^{2} \\
& \leq C\left(\int_{\Gamma_{\tau}}\left|e^{z t}\right|\left|\left(z_{1}^{\alpha}+\lambda_{j}\right)^{-1}\right|\left|z_{2}\right|^{-\gamma}\left|\frac{z \tau}{e^{z \tau}-1}\right||d z|\right)^{2}
\end{aligned}
$$

It is easy to see that $\left|\frac{z \tau}{e^{z \tau}-1}\right| \leq C$ for $z \in \Gamma_{\tau}$ and, by the Lemma $2.3,\left|\left(z_{1}^{\alpha}+\lambda_{j}\right)^{-1}\right| \leq\left(\left|z_{1}\right|^{\alpha}+\lambda_{j}\right)^{-1}$ for $z \in \Gamma_{\tau}$. Hence we obtain from (51) and noting that $z_{1} \sim z$ and $z_{2} \sim z$ on $\Gamma_{\tau}$, with some constants $\beta \in(0,1)$ and $c>0$,

$$
\begin{aligned}
\left\|E_{\tau}(t) e_{j}\right\|^{2} & \leq C\left(\left.\int_{\Gamma_{\tau}}\left|e^{z t}\right|\left(\left|z_{1}\right|^{\alpha}+\lambda_{j}\right)^{-1}|| z_{2}\right|^{-\gamma}|d z|\right)^{2} \leq C\left(\left.\int_{\Gamma_{\tau}}\left|e^{z t}\right|\left(|z|^{\alpha}+\lambda_{j}\right)^{-1}|| z\right|^{-\gamma}|d z|\right)^{2} \\
& \leq C\left[\int_{0}^{\tau^{-1}} e^{-c r t} r^{-\beta} d r\right]\left[\int_{0}^{\tau^{-1}} e^{-c r t} r^{\beta-2 \gamma}\left(r^{\alpha}+\lambda_{j}\right)^{-2} d r\right] \\
& \leq C t^{\beta-1}\left[\int_{0}^{\tau^{-1}} e^{-c r t} r^{\beta-2 \gamma}\left(r^{\alpha}+\lambda_{j}\right)^{-2} d r\right]
\end{aligned}
$$

Hence, we have, noting that $\lambda_{j} \sim j^{2 / d}, d=1,2,3$,

$$
\begin{aligned}
\mathbb{E}\|I I\|^{2} & \leq C \int_{0}^{t_{n}} t^{\beta-1}\left[\int_{0}^{\tau^{-1}} e^{-c r t} \sum_{j=M+1}^{\infty} r^{\beta-2 \gamma}\left(r^{\alpha}+\lambda_{j}\right)^{-2} d r\right] d t \\
& \leq C \int_{0}^{t_{n}} t^{\beta-1}\left[\int_{0}^{\tau^{-1}} e^{-c r t} \sum_{j=M+1}^{\infty} \frac{r^{\beta-2 \gamma}}{\left(r^{d \alpha / 2}+j\right)^{4 / d}} d r\right] d t
\end{aligned}
$$


Since $\sum_{j=M+1}^{\infty} \frac{1}{j^{4 / d}} \leq C \int_{M+1}^{\infty} \frac{1}{x^{4 / d}} \leq C \frac{1}{M^{4 / d-1}}$ for $d<4$, we have

$$
\begin{aligned}
\mathbb{E}\|I I\|^{2} & \leq C \int_{0}^{t_{n}} t^{\beta-1}\left[\int_{0}^{\tau^{-1}} e^{-c r t} \frac{r^{\beta-2 \gamma}}{\left(r^{d \alpha / 2}+M\right)^{4 / d-1}} d r\right] d t \\
& \leq C \int_{0}^{\tau^{-1}}\left[\int_{0}^{t_{n}} t^{\beta-1} e^{-c r t} d t\right] \frac{r^{\beta-2 \gamma}}{\left(r^{d \alpha / 2}+M\right)^{4 / d-1}} d r \\
& \leq C \int_{0}^{\tau^{-1}} r^{-\beta} \frac{r^{\beta-2 \gamma}}{\left(r^{d \alpha / 2}+M\right)^{4 / d-1}} d r \leq C \int_{0}^{\tau^{-1}} \frac{r^{-2 \gamma}}{\left(r^{d \alpha / 2}+M\right)^{4 / d-1}} d r \\
& \leq C \int_{0}^{\tau^{-1}} \frac{r^{-2 \gamma}}{\left(r^{d \alpha / 2}\right)^{4 / d-1}} d r \leq C \tau^{2(\alpha+\gamma)-1-d \alpha / 2} .
\end{aligned}
$$

Together these estimates complete the proof of Theorem 3.1.

\section{Numerical experiments}

Now we present some numerical results for the time-fractional stochastic PDE (1) with $0<\alpha<1$ and $0 \leq \gamma \leq 1$ on the unit interval $D=(0,1)$, in order to illustrate the error estimates in Theorems 2.8 and 3.1. For simplicity, we only consider the experimentally determined temporal convergence rates of the proposed numerical methods. More numerical simulations for the stochastic time fractional diffusions can be found in Jin et al. [21].

First, we briefly discuss the implementation of the noise term $W(t)$ [39]. Recall the Fourier expansion of the Wiener process $W(x, t)$

$$
W(x, t)=\sum_{\ell=1}^{\infty} e_{\ell}(x) \beta_{\ell}(t),
$$

where $\beta_{\ell}, \ell=1,2,3, \ldots$ are i.i.d. Brownian motions, and $e_{\ell}$ are the eigenfunctions of the negative Laplacian $A$. In particular, in the one-dimensional case, we have $e_{\ell}(x)=\sqrt{2} \sin (\ell \pi x), \ell=1,2,3, \ldots$ Thus the $L^{2}$-projection $P_{h} W(t)$ is given by

$$
\left(P_{h} W(t), \chi\right)=\sum_{\ell=1}^{\infty} \beta_{\ell}(t)\left(e_{\ell}, \chi\right) \approx \sum_{\ell=1}^{L} \beta_{\ell}(t)\left(e_{\ell}, \chi\right), \quad \forall \chi \in S_{h},
$$

where we have truncated the sum to $L$ terms. Recall that $\beta_{\ell}(t)$ are mutually independent standard realvalued Brownian motions and therefore the increments $\Delta \beta_{\ell}^{k}$ are given by

$$
\Delta \beta_{\ell}^{k}=\beta_{\ell}\left(t_{k}\right)-\beta_{\ell}\left(t_{k-1}\right) \sim \sqrt{\tau} \mathcal{N}(0,1),
$$

where $\mathcal{N}(0,1)$ denotes the standard Gaussian random variable. Further, $P_{h} d W\left(x, t_{k}\right)$ can be approximated by the backward difference

$$
P_{h} d W\left(x, t_{k}\right) \approx \frac{P_{h} W\left(t_{k}\right)-P_{h} W\left(t_{k-1}\right)}{\tau} .
$$

Thus the noise term ${ }_{0} I_{t}^{\gamma} d W\left(x, t_{n}\right)$ is approximated by

$$
{ }_{0} I_{t}^{\gamma} d W\left(x, t_{n}\right) \approx \tau^{\gamma} \sum_{k=1}^{n} \beta_{n-k}^{(-\gamma)}\left[\sum_{\ell=1}^{L} e_{\ell} \frac{\Delta \beta_{\ell}^{k}}{\tau}\right] .
$$

Throughout the experiments, we take initial data $u_{0}=0$ to focus on the effect of the noise $W$. In our computation, we divide the unit interval $D=(0,1)$ into $M$ equally spaced subintervals, with a mesh size 
$h=1 / M$ and we use the linear finite element method for the spatial discretization. We fix the time step size $\tau$ at $\tau=t / N$, where $t$ is the time of interest. All the results are computed with 100 trajectories.

The numerical results for various combinations of the fractional orders $\alpha$ and $\gamma$ are given in Table 1, where $t$ is fixed at $t=10^{-2}$ and $M=100$, and the reference solution is computed with $N=3200$. In the table, the numbers in the bracket in the last column denote the theoretical rates predicted by Theorems 2.8 and 3.1, which is $O\left(\tau^{\alpha+\gamma-\frac{1}{2}-\alpha d / 2-\epsilon}\right)$ with $d=1$ and $\epsilon>0$. The empirical convergence rates are in excellent agreement with the theoretical predictions, fully confirming the error estimates, despite the number of random realizations for computing the expectation is quite small.

Table 1: The $L^{2}(\Omega ; H)$ error at $t=10^{-2}$.

\begin{tabular}{|c|c|ccccc|c|}
\hline$\gamma$ & $\alpha \backslash N$ & 10 & 20 & 40 & 80 & 160 & rate \\
\hline 0.0 & 1 & $6.10 \mathrm{e}-2$ & $5.20 \mathrm{e}-2$ & $4.35 \mathrm{e}-2$ & $3.66 \mathrm{e}-2$ & $2.94 \mathrm{e}-2$ & $0.26(0.25)$ \\
\hline 0.2 & 0.8 & $1.94 \mathrm{e}-3$ & $1.43 \mathrm{e}-3$ & $1.04 \mathrm{e}-3$ & $7.73 \mathrm{e}-4$ & $5.45 \mathrm{e}-4$ & $0.45(0.45)$ \\
\hline 0.5 & 0.5 & $1.38 \mathrm{e}-3$ & $1.05 \mathrm{e}-3$ & $8.04 \mathrm{e}-4$ & $6.12 \mathrm{e}-4$ & $4.39 \mathrm{e}-4$ & $0.41(0.38)$ \\
\hline 0.8 & 0.2 & $1.14 \mathrm{e}-5$ & $9.41 \mathrm{e}-6$ & $7.55 \mathrm{e}-6$ & $5.95 \mathrm{e}-6$ & $4.48 \mathrm{e}-6$ & $0.34(0.30)$ \\
\hline
\end{tabular}

\section{Concluding remarks}

In this work, we have developed a numerical scheme for approximating the stochastic time-fractional diffusion problem driven by integraterd space-time white noise. The time fractional derivative is approximated by using the L1 scheme and the fractional integral is approximated by using the first order convolution quadrature formula. The space-time white noise is approximated by using the Euler method in time and the truncated series in space. The spatial variable is approximated by using a standard finite element method. Based on the new developed convolution expression of the approximate solution, we obtain the strong error estimates of the fully discrete scheme for stochastic subdiffusion problem in multidimensional case. In our future work, we shall consider the numerical approximations for the semilinear stochastic subdiffusion problems driven by integrated space-time white noise.

\section{Acknowledgments}

The authors are grateful to the Reviewers and the Associate Editor for their helpful comments.

\section{References}

[1] E. E. Adams and L. W. Gelhar, Field study of dispersion in a heterogeneous aquifer: 2. Spatial moments analysis, Water Res. Research, 28 (1992), pp. 3293-3307.

[2] V. V. Anh, N. N. Leonenko, and M. Ruiz-Medina, Space-time fractional stochastic equations on regular bounded open domains, Fract. Calc. Appl. Anal., 19(2016), 1161-1199.

[3] L. Chen, Nonlinear stochastic time-fractional diffusion equations on R: moments, Hölder regularity and intermittency, Trans. Amer. Math. Soc., pages in press, arXiv:1410.1911, 2016.

[4] L. Chen, Y. Hu, and D. Nualart, Nonlinear stochastic time-fractional slow and fast diffusion equations on Rd, preprint, arXiv:1509.07763, 2015.

[5] Z.-Q. Chen, K.-H. Kim, and P. Kim, Fractional time stochastic partial differential equations, Stochastic Process. Appl., 125(2015), pp. 1470-1499.

[6] G. Da Prato and J. Zabczyk, Stochastic Equations in Infinite Dimensions, volume 152 of Encyclopedia of Mathematics and its Applications, Cambridge University Press, Cambridge, second edition, 2014.

[7] W. Deng and J. S. Hesthaven, Local discontinuous Galerkin methods for fractional ordinary differential equations, BIT, 55(2015), pp. 967-985.

[8] K. Diethelm, An algorithm for the numerical solution of differential equations of fractional order, Electronic Transactions on Numerical Analysis, 5(1997), pp. 1-6.

[9] M. Foondun, J. Mijena, and E. Nane, Non-linear noise excitation for some space-time fractional stochastic equations in bounded domains, Fract. Calc. Appl. Anal., 19(2016), 1527-1553.

[10] N. J. Ford and K. Pal and Y. Yan, An algorithm for the numerical solution of space-fractional partial differential equations, Computational Methods in Applied Mathematics, 15(2015), pp. 497-514. 
[11] N. J. Ford, J. Xiao and Y. Yan, A finite element method for time-fractional partial differential equations, Fract. Calc. Appl. Anal., 14(2011), pp. 454-474.

[12] G.-H. Gao, Z.-Z. Sun and H.- W. Zhang, A new fractional numerical differentiation formula to approximate the Caputo fractional derivative and its applications, J. Comput. Phys., 259 (2014), 33-50.

[13] R. Gorenflo and F. Mainardi, Random walk models for space fractional diffusion processes, Fract. Calc. Appl. Anal., 1(1998), pp. 167-191.

[14] M. Gunzburger, B. Li, and J. Wang, Convergence of finite element solution of stochastic partial integral-differential equations driven by white noise, Numerische Mathematik, 141(2019), pp. 1043-1077.

[15] M. Gunzburger, B. Li, and J. Wang, Sharp convergence rates of time discretization for stochastic time-fractional pdes subject to additive space-time white noise, Math. Comp, 88(2019), pp. 1715-1741.

[16] Y. Hatano and N. Hatano, Dispersive transport of ions in column experiments: An explanation of long-tailed profiles, Water Res. Research, 34(1998), pp. 1027-1033.

[17] B. Jin, R. Lazarov and Z. Zhou, Error estimates for a semidiscrete finite element method for fractional order parabolic equations, SIAM J. Numer. Anal., 51(2013), pp. 445-466.

[18] B. Jin, R. Lazarov and Z. Zhou, An analysis of the L1 scheme for the subdiffusion equation with nonsmooth data, IMA J. of Numer. Anal., 36(2016), pp. 197-221.

[19] B. Jin, B. Li, and Z. Zhou, An analysis of the Crank-Nicolson method for subdiffusion, IMA J. of Numer. Anal., 38(2018), pp. 518-541.

[20] B. Jin, B. Li, and Z. Zhou, Correction of high-order BDF convolution quadrature for fractional evolution equations, SIAM J. Sci. Comput., 39(2017), pp. A3129-A3152.

[21] B. Jin, Y. Yan, and Z. Zhou, Numerical approximation of stochastic time-fractional diffusion, ESAIM: M2AN., 53(2019), pp. 1245-1268.

[22] M. Kovács and J. Printems, Strong order of convergence of a fully discrete approximation of a linear stochastic Volterra type evolution equation, Math. Comp., 83(2014), pp. 2325-2346.

[23] M. Kovács and J. Printems, Weak convergence of a fully discrete approximation of a linear stochastic evolution equation with a positive-type memory term, J. Math. Anal. Appl., 413(2014), pp. 939-952.

[24] Y. Li, Y. Wang, and W. Deng, Galerkin finite element approximations for stochastic space-time fractional wave equations, SIAM Journal on Numerical Analysis, 55(2017), pp. 3173-3202.

[25] Y. Lin and C. Xu, Finite difference/spectral approximations for the time-fractional diffusion equation, J. Comput. Phys., 225(2007), pp. 1533-1552.

[26] W. Liu, M. Röckner, and J. L. d. Silva, Quasi-linear (stochastic) partial differential equations with time-fractional derivatives, SIAM J. Math. Anal., 50(2018), pp. 2588-2607.

[27] Ch. Lubich, I.H. Sloan and V. Thomée, Nonsmooth data error estimate for approximations of an evolution equation with a positive-type memory term, Math. Comp., 65(1996), pp. 1-17.

[28] W. McLean and K. Mustapha, Time-stepping error bounds for fractional diffusion problems with non-smooth initial data, J. Comput. Phys., 293(2015), pp. 201-217.

[29] J. B. Mijena and E. Nane, Space-time fractional stochastic partial differential equations, Stochastic Process. Appl., 125(2015), pp. 3301-3326.

[30] J. B. Mijena and E. Nane, Intermittence and space-time fractional stochastic partial differential equations, Potential Anal., 44(2016), 295-312.

[31] K. Mustapha and W. McLean, Uniform convergence for a discontinuous Galerkin, time-stepping method applied to a fractional diffusion equation, IMA J. of Numer. Anal., 32(2012), pp. 906-925.

[32] R. Nigmatulin, The realization of the generalized transfer equation in a medium with fractal geometry, Phys. Stat. Sol. B, 133(1986), pp. 425-430.

[33] K. Sakamoto and M. Yamamoto, Initial value/boundary value problems for fractional diffusion-wave equations and applications to some inverse problems, J. Math. Anal. Appl., 382(2011), pp. 426-447,

[34] M. Stynes, Too much regularity may force too much uniqueness, Fractional Calculus and Applied Analysis, 19(2016), $1554-1562$.

[35] M. Stynes, E. O'riordan and J. L. Gracia, Error analysis of a finite difference method on graded meshes for a timefractional diffusion equation, SIAM J. Numer. Anal., 55(2017), pp. 1057-1079.

[36] Y. Yan, M. Khan and N. J. Ford, An analysis of the modified scheme for the time-fractional partial differential equations with nonsmooth data, SIAM J. Numer. Anal., 56(2018), 210-227.

[37] Y. Yan, K. Pal and N. J. Ford, Higher order numerical methods for solving fractional differential equations, BIT Numer. Math, 54(2014), pp. 555-584

[38] V. Thomée, Galerkin Finite Element Methods for Parabolic Problems, Springer - Verlag, Berlin, 2007.

[39] Y. Yan, Galerkin fnite element methods for stochastic parabolic partial differential equations, SIAM J. Numer. Anal., 43(2005), 1363-1384.

[40] M. Zayernouri, M. Ainsworth, and G. E. Karniadakis, A unified Petrov-Galerkin spectral method for fractional pdes, Comput. Methods Appl. Mech. Engrg., 283(2015), pp. 1545-1569.

[41] F. Zeng, C. Li, F. Liu and I. Turner, The use of finite difference/element approaches for solving the time-fractional subdiffusion equation, SIAM J. Sci. Comput., 35(2013), pp. A2976-A3000.

[42] F. Zeng, Z. Zhang, and G. E. Karniadakis, Second-order numerical methods for multi-term fractional differential equations: Smooth and non-smooth solutions, Computer Methods in Applied Mechanics and Engineering, 327(2017), pp. 478-502. 